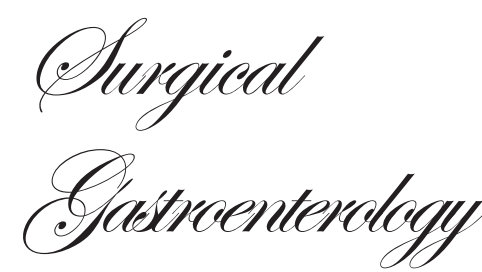

\title{
Role of diagnostic laparoscopy in chronic and recurrent abdominal pain
}

\author{
Musharraf Husain, Praveendra Kumar Sachan, Sabina Khan, \\ Lovneesh Lama, Rehan N Khan
}

\begin{abstract}
Department of Surgery

Hamdard Institute of Medical

Sciences and Research,

Jamia Hamdard, New Delhi, India

\section{Correspondence:}

Dr Musharraf Husain

Email:drmhusain1@ rediffmail.com

Background: Chronic abdominal pain is a very common condition presenting to a general surgeon. It is defined as pain lasting for more than 3 months. Sometimes, the diagnosis cannot be established even with the help of advanced radiological investigations. This study aims to define the role of laparoscopy in diagnosing the cause of chronic abdominal pain and thus enabling a definitive management.

Methods: The study included 52 patients admitted to the Department of Surgery, Himalayan Institute of Medical Sciences, Dehradun, India for chronic abdominal pain of unknown origin. All patients underwent diagnostic laparoscopy with methodical inspection of the whole abdomen.

Results: Diagnosis was established in $86.5 \%$ of cases either by direct visualization or histopathological examination. The most common pathology was chronic appendicitis (19\%) followed by adhesions (17.3\%) and peritoneal tuberculosis (15.3\%). A therapeutic procedure was done in $46 \%$ of cases during laparoscopy. No major complication was reported during the study.

Conclusions: Laparoscopy is a safe and effective method to diagnose the cause of chronic abdominal pain in cases where other non-invasive methods prove unreliable.
\end{abstract}

$K E Y W O R D S$ : diagnostic laparoscopy, chronic abdominal pain, chronic appendicitis

\section{Introduction}

Chronic abdominal conditions represent a major group of cases presenting to a general surgeon. Mostly, a diagnosis can be made by clinical examination alone or with the help of investigations such as ultrasound or computed tomography (CT) scan. But there are a large number of patients in whom the diagnosis cannot be made even with these highly sensitive and advanced modalities. It is thus a great challenge for the surgeon to diagnose accurately and decide the best treatment modality. ${ }^{1}$
Laparoscopy is as much a surgical procedure as exploratory laparotomy and, very often, just as informative. Apart from visualizing a large part of the abdominal cavity a precise targeted biopsy, fine-needle aspiration cytology or fluid analysis can also be done.

Laparoscopy offers a distinct advantage over ultrasound or CT scan as it is capable of detecting lesions $<5 \mathrm{~mm}$ in size especially peritoneal metastasis, which cannot be detected by these investigations. Laparoscopy-guided biopsy provides a 
larger histopathological specimen as compared to percutaneous biopsy. It is more accurate than the radiological-guided biopsy and can provide a diagnosis in $85 \%-95 \%$ of patients. ${ }^{2}$ Another advantage is that haemostasis can be achieved accurately under direct visualization with the help of cautery. However, it has certain disadvantages also, such as a higher cost, longer operative time and requires general anaesthesia.

Chronic or recurrent abdominal pain is defined as pain lasting for $>3$ months. It is a very common medical problem with a wide variety of associated abdominal diseases.

The aim of this study was to know the accuracy of laparoscopy in diagnosing patients having chronic abdominal pain of unknown origin and providing them the best possible treatment.

\section{Method and materials}

This study was conducted in the Department of Surgery, Himalayan Institute of Medical Sciences, Dehradun, India. It was a prospective study comprising 52 patients, conducted over a period of one-and-a-half year. All the patients admitted with complaints of chronic abdominal pain of unknown origin were included in the study. The inclusion criteria were patients with normal or inconclusive investigation for pain abdomen and in whom no medical cause of pain could be found. The exclusion criteria were patients with known medical cause of pain abdomen, functional bowel disease, clinically detectable ascites, uncorrectable coagulopathy and patients with severely decompensated cardiorespiratory system.

All patients were subjected to a thorough general physical and systemic examination along with gynaecological examination (in women). Routine investigations such as complete blood count, coagulation profile, urine examination, renal function test, X-ray chest and abdomen and ultrasound whole abdomen were performed in all the patients. Upper gastrointestinal (GI) endoscopy was performed in patients with upper abdominal pain only. CT scan was done in 32 patients while 20 patients refused to take the test because of financial constraints.

Diagnostic laparoscopy was performed in all the 52 patients. Methodical inspection of all the quadrants of the abdomen starting from the pelvis was done. Targeted fine-needle aspiration cytology and fluid aspiration from the peritoneal cavity was done as and when indicated. Only a single shot of second-generation cephalosporins was given at the time of induction of anaesthesia. Most of the patients were discharged on postoperative day 2. A followup examination was done after 6 months to evaluate the outcome of all these cases.

\section{Results}

The total number of patients in our study was 52, out of which 29 were women and 23 were men (mean age: 33 years; range 18-63 years). Most of the patients belonged to the age group of 31-40 years. A diagnosis was established in 45 of 52 patients and various therapeutic procedures were performed according to the diagnosis. In our study, 34 (66\%) patients had inflammatory pathology while neoplastic pathology was found in $12(23 \%)$ patients. The cause of pain could not be ascertained in 5 patients while 1 patient had a miscellaneous pathology. The average duration of surgery was 48 minutes (range 15-130 minutes) and the average length of hospital stay was 2.3 days (range 1-9 days)

The most common diagnosis for chronic abdominal pain was chronic appendicitis in 10 (19\%) patients. A diagnosis was made during laparoscopy by finding either appendicoliths or thickened appendix with surrounding adhesions. This was followed by adhesions in $9(17.3 \%)$ patients and peritoneal tuberculosis in $8(15.3 \%)$ patients. The patients with peritoneal tuberculosis presented with grumbling abdominal pain, had detectable ascites on ultrasound examination and found to have rolled-up omentum or peritoneal deposits during diagnostic laparoscopy. There were four cases of carcinoma gallbladder and three cases of metastatic deposits in the liver; all these patients refused CT scan examination. The primary could be detected in all 3 patients with liver metastasis. Three cases each of cirrhosis of the liver and endometriosis were detected. Two cases of ovarian cyst (missed by ultrasound examination) were diagnosed on laparoscopy. There were two unusual cases-one of ectopic tubal pregnancy and other of Crohn's disease. No pathology could be detected in 5 patients even after laparoscopy. The diagnosis could not be established in 2 patients with ascites even after biopsy examination, although laparoscopic findings were suggestive of tuberculosis (Table 1).

Diagnoses of all of the above conditions were confirmed either by direct visualization or by taking biopsy in all cases of malignancy and tuberculosis. Therapeutic procedures were carried out simultaneously in $46 \%$ of patients - appendectomy (10), adhesiolysis (9), fulguration for endometriosis (3) and ovarian cystectomy (2). Therapeutic laparotomy was performed in 3 patients. Gastrojejunostomy was done for gastric outlet 
Table 1: Pathological diagnosis and their treatment

\begin{tabular}{|c|c|c|c|}
\hline Pathology & $\mathrm{N}=52$ & $\%$ & Treatment \\
\hline Chronic appendicitis & 10 & 19 & Appendectomy \\
\hline Peritoneal tuberculosis & 8 & 15.3 & Anti-tubercular treatment \\
\hline Adhesion & 9 & 17.3 & Adhesiolysis \\
\hline Carcinoma gallbladder & 4 & 7.6 & $\begin{array}{l}\text { Gastrojejunostomy (1) } \\
\text { Radical choleccystectomy (1) } \\
\text { Referred for chemotherapy (2) }\end{array}$ \\
\hline $\begin{array}{l}\text { Metastatic disease of } \\
\text { the liver }\end{array}$ & 3 & 5.7 & Referred for chemotherapy \\
\hline Cirrhosis of the liver & 3 & 5.7 & Referred to gastroenterology \\
\hline Endometriosis & 3 & 5.7 & Fulguration \\
\hline Ovarian cyst & 2 & 3.8 & Ovarian cystectomy \\
\hline $\begin{array}{l}\text { Genitourinary } \\
\text { tuberculosis }\end{array}$ & 2 & 3.8 & Anti-tubercular treatment \\
\hline Salpingitis & 1 & 1.9 & Medical treatment \\
\hline $\begin{array}{l}\text { Tubal ectopic } \\
\text { pregnancy }\end{array}$ & 1 & 1.9 & Salpingostomy \\
\hline Crohn's disease & 1 & 1.9 & Resection and anastomosis \\
\hline Normal study & 5 & 9.6 & Medical treatment (1) \\
\hline
\end{tabular}

obstruction in an advanced case of carcinoma gallbladder; radical cholecystectomy was performed for stage II carcinoma gallbladder while resection and anastomosis was done for stricture in the terminal ileum because of Crohn's disease.

Ascites was present in 20 patients, abdominal ultrasound examination was able to diagnose ascites in only 14 cases (sensitivity 70\%). Laparoscopy detected the cause of ascites in 18 patients with the help of direct visualization or histopathological examination. Ascites was present in eight cases of peritoneal tuberculosis, two cases of carcinoma gallbladder, three cases each of liver metastasis and cirrhosis of the liver, two cases of genitourinary tuberculosis and two cases of abdominal adhesions.

No major complications were reported. Only two cases developed subcutaneous emphysema, while one patient developed umbilical port site minor infection which was treated with antibiotics.

\section{Discussion}

Laparoscopy is an excellent diagnostic modality which is often underutilized due to risks inherent to surgical procedure. With the advances in technology and increasing expertise in laparoscopy, the safety of these procedures is established beyond doubt. It helps in making a diagnosis where all other methods fails. In the present study, the aetiology of chronic abdominal pain could be established in $86 \%$ of cases. In the same sitting, a definitive therapeutic procedure was performed in $44 \%$ of cases. Positive histopathological reports to support the diagnosis were obtained in $74 \%$ of cases.

Chronic appendicitis is a very common pathology missed by normal radiological investigations such as ultrasound and sometimes even on CT scan. The advantage of laparoscopy in these patients is that they can be provided therapy in the same setting. Kolts et al., ${ }^{3}$ in their study of 44 children with chronic lower abdominal pain, showed resolution of symptoms in $70.5 \%$ of cases after appendectomy. Similarly, in our series complete or partial relief was obtained in $77.7 \%$ of patients. Complete relief was labelled when a patient did not have any episode of pain after discharge from the hospital while partial relief was labelled when either episodes of pain were decreased in frequency or there was a decrease in the usage of analgesics.

The diagnosis of isolated peritoneal tuberculosis is difficult as the size of the tubercles is usually $<5 \mathrm{~mm}$, which are not detected on ultrasound examination or CT scan. Laparoscopy provides a specimen for definite histopathological diagnosis. Rodriquez de Lope et al. ${ }^{4}$ diagnosed 14 cases of tubercular ascites with the help of peritoneal biopsy. We had a total of eight cases of peritoneal tuberculosis that presented with ascites of unknown origin, detected on ultrasound examination. Laparoscopy confirmed the diagnosis with the help of gross appearance or peritoneal biopsy.

Paajanen et al. ${ }^{5}$ found adhesions to be the most important cause of chronic abdominal pain during diagnostic laparoscopy ( $85 \%$ of cases). However, in the present study, an adhesion was the cause of pain in only $17.3 \%$ of cases. All of them improved completely or partially after adhesiolysis during the follow-up period of 6 months. This can be explained on the basis of high rates of incidence of abdominal tuberculosis and gallbladder malignancy in this region, different inclusion and exclusion criteria and non-availability of CT scan for all the patients.

The diagnostic accuracy of laparoscopy is as high as $100 \%$ in diagnosing intra-abdominal malignancy. ${ }^{4}$ Similarly, we were also able to diagnose all seven cases of malignancy- 4 cases of carcinoma gallbladder and three having metastatic disease with unknown primary. A few of these cases could have been diagnosed by CT scan, if not refused by the patient; however, we could take the biopsy after localizing the lesions during laparoscopy.

The case for avoiding unnecessary laparotomy by various authors ranged from $49 \%$ to $85 \% .{ }^{6,7}$ In our study, we were able to avoid unnecessary laparotomy in $90 \%$ of patients. 
Therapeutic laparotomy was performed in three cases. Gastrojejunostomy was done for gastric outlet obstruction in an advanced case of carcinoma gallbladder, radical cholecystectomy was performed for stage II carcinoma gallbladder, resection anastomosis was done for the stricture in the terminal ileum because of Ccrohn's disease.

Salky and Edye ${ }^{8}$ were able to establish the aetiology in 201 (76\%) out of 387 patients undergoing diagnostic laparoscopy. A definitive therapeutic procedure was performed in 128 (48\%) patients. In our study, diagnosis was established in $86 \%$ of cases while the rate of definitive therapeutic procedure was $46 \%$.

In the present study, we had a total of 20 cases of ascites either diagnosed preoperatively by ultrasound examination or during laparoscopy. The cause of ascites could not be ascertained in two cases even with histopathological examination. But they appeared to be tuberculous on direct visualization; therefore, anti-tubercular treatment was started and both the patients responded well. They were categorized under the peritoneal tuberculosis group.

The rates of complication reported in the literature are as low as $<1 \%$. ${ }^{1}$ Similarly, no major complication was reported in our study.

There are two main limitations of our study-firstly, the patient group is small and secondly, CT scan could not be done in all the patients before diagnostic laparoscopy due to patients' refusal.
To conclude, diagnostic laparoscopy is a safe and effective procedure without any major risk or complications in evaluating patients with chronic abdominal pain. If done early in the course of the disease, it helps in reducing the hospital stay, treatment cost and morbidity to the patient.

\section{References}

1. Udwadia TE. Laparascopic surgery in developing countries. 1st ed. India: Jaypee Brothers Medical Publishers; 1997.

2. Jori GP, Peschle CL. Combined peritoneoscopy and liver biopsy in the diagnosis of hepatic neoplasm. Gastroenterology. 1972;63:1016-19.

3. Kolts RL, Nelson RS, Park R, Heikenen J. Exploratory laparoscopy for recurrent right lower quadrant pain in a pediatric population. Pediatr Surg Int. 2006;22:247-9.

4. Rodriquez de Lope C, San Miquel Joglar G, Pons Romero F. Laparascopic diagnosis of tuberculous ascites. Endoscopy. 1982;14:178-9.

5. Paajanen H, Julkunen K, Waris H. Laporoscopy in chronic abdominal pain: a prospective nonrandomized long-term followup study. J Clin Gastroenterlogy. 2005;39:110-14.

6. Easter DW, Cuschieri A, Nathanson LK, Lavelle-Jones M. The utility of diagnostic laparoscopy for abdominal disorders. Audit of 120 patients. Arch Surg. 1992;127:379-83.

7. Vander Velpen GC, Shimi SM, Cuschieri A. Diagnostic yield and management benefit of laparoscopy: a prospective audit. Gut. 1994;35:1617-21.

8. Salky BA, Edye MB. The role of laparoscopy in the diagnosis and treatment of abdominal pain syndromes. Surg Endosc. 1998;12:911-14 